\title{
La traduzione catalana del commento di Bernardo Ilicino ai Triumphi del Petrarca: alcune novità a proposito del modello italiano*
}

\section{Leonardo Francalanci}

Universitat de Girona

leonardo.francalanci@udg.edu

\section{Abstract}

In questo articolo si approfondiscono alcuni aspetti filologici della traduzione catalana tardoquattrocentesca del commento di Bernardo Ilicino ai Triumphi del Petrarca. L'analisi testuale consente, in primo luogo, di identificare la redazione a partire dalla quale fu realizzata la traduzione (si tratta del testo trasmesso dall'incunabolo veneziano del 1478, contenente sia Triumphi che il Rerum Vulgarium Fragmenta) e, in secondo luogo, grazie anche all'analisi delle traduzioni castigliane cinquecentesche, di stabilirne una datazione più stretta di quelle proposte sinora (post quem 1478 - ante quem 1512).

Parole chiave: commenti quattrocenteschi ai Triumphi, Bernardo Ilicino, Triumphi, Francesco Petrarca.

\section{Abstract}

In this article we perform an in-depth study of some philological aspects of the late $15^{\text {th }}$ century Catalan translation of Bernardo Ilicino's commentary on the Triumphs of Petrarca. The textual analysis allows us, first, to identify the wording upon which our translation was based (this is the text of the Venetian incunabulum of 1478 which contained both the Triumphs and the Rerum Vulgarium Fragmenta), and, second, thanks also to the analysis of the $16^{\text {th }}$ century Spanish translations, to provide a more precise dating (post quem 1478 - ante quem 1512).

Key words: late $15^{\text {th }}$ century commentaries to Triumphs, Bernardo Ilicino, Triumphs, Francesco Petrarca. 
La traduzione catalana del commento di Bernardo Ilicino ai Triumphi di Francesco Petrarca, ${ }^{1}$ di autore anonimo ${ }^{2}$ e di datazione incerta, ${ }^{3}$ è trasmessa da un unico manoscritto oggi diviso in due tomi, conservati rispettivamente a Parigi (BNF, ms. Esp. 534) e a Barcellona (Ateneu, ms. II). La storia del codice ci è oscura fino alla fine del XVI secolo, a cui risale l'ex libris al margine superiore del f. I del primo tomo "Est d[omini] Jo[annes] Vich epi[scopi] Maiorice[nsis]", dal quale si deduce che appartenne al valenzano Joan Vich Manrique (15301611), vescovo di Maiorca tra il 1573 ed il 1604, anno in cui divenne arcivescovo di Tarragona. ${ }^{4}$ Successivamente il codice passò al monastero della Murta nel Regno di Valenza, dove rimase fino all'Ottocento, quando entrò in possesso del bibliofilo Miquel Victorià Amer. Alla morte di questi, essendone stata messa in vendita la biblioteca, fu diviso in due tomi, il primo dei quali entrò alla Biblioteca Nazionale di Parigi il 21 aprile 1897, e il secondo alla biblioteca dell'Ateneu di Barcellona. ${ }^{5}$

Pur trattandosi di un'opera da sempre piuttosto nota alla critica, nonché di un importante tassello per lo studio della presenza e soprattutto dell'assimilazione dell'opera petrarchesca in ambito catalano, la mancanza di dati, assieme all'impossibilità di stabilirne una datazione certa, ha fatto si che pochi studiosi se ne siano occupati a fondo, e che molte questioni inerenti a questa traduzione restino ancora senza risposta. Del resto, una situazione analoga, anche se per motivi in parte diversi, interessa il commento dell'Ilicino anche in ambito italiano: pochi, infatti, se ne sono occupati, e chi vi si è cimentato lo ha fatto generalmente in modo superficiale, vedendovi solo una delle numerose manifestazioni della fortuna tardo-quattrocentesca del-

1. La particolarità di questa traduzione risiede nella scelta dell'autore di intervenire soltanto sul testo del commento, lasciando i versi poetici che accompagna in italiano.

2. Il manoscritto fu composto plausibilmente da un autore valenzano. Tale possibilità fu già segnalata, a partire da due esempi — ma ve ne sono molti altri- nei quali appare la tipica grafia -ch-dell'apichat (chiquet infant, f. 17v; Syl-la chiquet, f. 39r), dal Massó Torrents nella sua descrizione codicologica del manoscritto (1902). La scritta "Petrarca comentat en Valencià», che troviamo sulla coperta del tomo parigino, risale invece al XVIII-XIX sec, e testimonia che la provenienza valenzana era già evidente prima di questo intervento. Jaume MASSÓ TORRENTS, Biblioteca del Ateneo Barcelonés. Cataleg dels manuscrits, Barcelona, 1902, p. 56-61.

3. La critica concorda nel datare il codice genericamente alla seconda metà del Quattrocento, anche se qualche studioso, come il Romano, non esclude l'eventualità che possa risalire ai primi del XVI secolo. David RomAnO, «Acerca del ms. del Ateneo Barcelonés de los Triomfi de Petrarca», in Boletín de la Real Academia de Buenas Letras de Barcelona, Barcelona (1987-88), p. 8-9.

4. Dato che la nota di possesso lo identifica come titolare della sede majorchina, il manoscritto potrebbe essere entrato in suo possesso durante questo lasso di anni.

5. Buona parte degli studiosi ritengono che il codice sia stato diviso alla fine del XIX secolo, poco prima dell'acquisto da parte della Biblioteca Nazionale di Parigi. Legato forse da sempre come volume unico, potrebbe invece essere stato progettato in due tomi già in fase di scrittura. Il testo, infatti, appare volutamente interrotto dopo il secondo capitolo del T. Mortis, metà naturale del poema: l'ultima carta del codice parigino (c. 250r) è in bianco, tranne la prima riga "l'any mil CCC LXXIIII", che corrisponde al richiamo l'any presente nal margine inferiore dell'ultima carta del fascicolo precedente (c. $249 \mathrm{v})$, seguita soltanto da un tratto obliquo. 
l'opera del Petrarca. L'obbiettivo principale di questo articolo sarà dunque quello di colmare alcune di queste lacune, la più importante delle quali - poiché propedeutica alle altre- è rappresentata certamente dal problema della datazione dell'opera.

Troppo spesso, mi sembra, si commette l'errore di sottovalutare l'importanza del commento dell'Ilicino, soprattutto dal punto di vista socio-culturale: il commento del senese Bernardo Lapini da Montalcino, detto Ilicino, ${ }^{6}$ composto molto probabilmente nel periodo $1468-1469,{ }^{7}$ e stampato per la prima volta a Bologna nel 1475 da Hannibal Malpiglius e Sigismundus de Libris, rappresenta infatti, senza ombra di dubbio, il più celebre e il più diffuso dei commenti quattrocenteschi ai Triumphi del Petrarca. ${ }^{8}$ Ciò significa, evidentemente, che nella seconda metà del Quattrocento, e in buona misura fino alla prima metà del Cinquecento, quando sarà definitivamente sostituito da quello di Alessandro Vellutello (1525), chi leggeva i Triumphi del Petrarca quasi certamente lo faceva attraverso il commento iliciniano e, come dimostra la nostra traduzione catalana, non soltanto in ambito italiano. ${ }^{9}$ Purtroppo, l'enorme diffusione del testo non corrisponde però all'interesse suscitato nella critica, e col passare del tempo la fama dell'Ilicino, contrariamente a quella del Petrarca, venne progressivamente meno. Non si può però imputarne la responsabilità soltanto ai limiti, tutto sommato

6. Anche se tralasceremo in questa sede la biografia dell'Ilicino, converrà comunque ricordarne due aspetti fondamentali: innanzitutto dovremo tenere presente che il nostro autore, "medicus et philosophus discipulus», formatosi presso lo Studio senese facente capo al Dati, non era propriamente né un umanista né un intellettuale; in secondo luogo sarà utile osservare che l'Ilicino, figlio di quel «maistro Pietro da Montalcino» medico alla corte milanese di Filippo Maria Visconti (1412-1447) e autore di un commento oggi perduto ai sonetti del Petrarca, aveva dunque ereditato dal padre sia la vocazione medica che quella letteraria.

7. I due estremi cronologici sicuri entro cui si colloca la stesura del commento sono infatti post 1466, morte di Francesco Sforza, ricordata nel commento al v. 92 del primo capitolo del T. Mortis, e ante 14 aprile 1471, data in cui Borso d'Este, dedicatario del commento, venne elevato al ducato di Ferrara: il commento lo indica infatti soltanto come "Mutinae Ducem». All'epoca della stesura del suo commento ai Triumphi, Bernardo Ilicino ricopriva la carica di medico presso la corte estense a Ferrara.

8. Il commento dell'Ilicino ebbe, dal momento della sua prima apparizione a stampa nel 1475, e per i circa 50 anni successivi, un'enorme fortuna, finché, a partire dal primo Cinquecento, con l'edizione del poema curata dal Bembo, basata sugli autografi petrarcheschi, il testo divenne rapidamente obsoleto. Il corpus ammonta infatti a ben 24 edizioni a stampa (tra il 1475 e il 1522) e ad almeno nove manoscritti, tutti databili all'ultimo quarto del XV secolo circa, cioè contemporanei (in qualche caso forse addirittura antecedenti) al primo incunabolo bolognese del 1475 e dunque vicinissimi alla data di composizione dell'opera, segno inequivocabile di una straordinaria, quanto precocissima, fortuna letteraria.

9. Oltre alla traduzione catalana, oggetto di questo articolo, ne esistono infatti una in francese (BNF, mss., Français 223 e 549; questo codice risulta particolarmente interessante perché presenta, come la nostra traduzione catalana, i versi in italiano e il commento tradotto in francese) e due in castigliano (Antonio de Obregón, 15121; Hernando Hozes del 15541), nonchè una latina, trasmessa però da un manoscritto d'origine italiana. 
non pochi, ${ }^{10}$ dell'esercizio iliciniano: l'oblio nel quale è caduto il commento dipende soprattutto dall'evoluzione del gusto letterario, che nel corso dei secoli ha finito per relegare i Triumphi, e a maggior ragione il commento che li accompagnava, in secondo piano rispetto ai Rerum Vulgarium Fragmenta.

Dal punto di vista filologico - non entreremo qui nel merito di quello letterario-, il testo iliciniano presenta alcune caratteristiche peculiari, la più importante delle quali, o almeno la più evidente, è la struttura stessa dell'opera. Come sappiamo, infatti, il Petrarca non ultimò mai il testo dei Triumphi, lasciando un'architettura globale del poema non definitiva, con molteplici redazioni di alcuni capitoli tutte equivalenti fra loro; questa situazione, oggi in buona parte risolta, era invece ancora nel Quattrocento una questione apertissima, e uno dei principali problemi che i commentatori dovevano risolvere era proprio quello di approntare un'edizione del testo poetico, stabilendo quali capitoli o lezioni mettere a testo e quali invece rifiutare. Uno dei luoghi più problematici, anche se non certo l'unico, era allora rappresentato dei primi due capitoli del T. Fame: Da poi che morte triumfo nel volto e Nel cor pien d'amarissima dolcezza. Come ho già dimostrato in un precedente articolo a proposito del commento iliciniano, ${ }^{11}$ se è vero che dal punto di vista prettamente letterario questa particolare realtà testuale può costituire un limite, risulta invece decisiva da quello filologico, poiché lo studio dell'ordine e della natura di questi due capitoli ci consente di individuare, per il testo del commento, tre fasi redazionali ed editoriali ben distinte, ${ }^{12}$ riassumibili in questi termini:

I ) Manoscritto Ital 397, $\alpha$ H. 3, 2 della Biblioteca Estense di Modena (molto probabilmente il manoscritto di dedica a Borso D'Este autorizzato dall'autore) e primo incunabolo bolognese del 1475: il T. Famae è costituito da tre capitoli, il primo dei quali è composto per i versi 1-22 $1 / 2$ da quelli di Nel cor pien d'amarissima dolcezza, mentre per i restanti da quelli di Da poi che morte, con il commento che segue questa distribuzione; questa è la sistemazione originaria del commento attribuibile all'Ilicino.

II ) Incunaboli 1478-1500: a partire dalla seconda edizione veneziana del 1478, e così per tutti i successivi incunaboli, il T. Famae presenterà invece quattro capitoli, Nel cor come primo e Da poi che morte come secondo, entrambi per esteso e corredati del relativo commento.

10. Si tratta infatti di un testo in prosa lunghissimo - a detta del Dionisotti «uno dei più ampi testi volgari quattrocenteschi»—, la cui abbondante farcitura retorico-didattica ne rende la lettura tutt'altro che piacevole. Carlo DiONISOTTI, «Fortuna del Petrarca nel Quattrocento», in Italia Medioevale e Umanistica, Padova: Antenore, XVII, 1974, p. 61-113.

11. Leonardo FranCALANCI, «Il commento di Bernardo Ilicino ai Triumphi del Petrarca: Alcune questioni di metodo", in Studi di Filologia Italiana, Firenze: Le Lettere, LXIV, 2006.

12. La particolare natura del corpus del commento ( 24 edizioni a stampa contro 9 testimoni manoscritti), fa sì che l'analisi filologica del commento dell'Ilicino verta principalmente sui numerosi testimoni a stampa, mentre la tradizione manoscritta, tranne il caso del codice estense autorizzato dall'autore, passa in secondo piano. 
III ) Edizioni successive a quella del 1501, questa compresa: la situazione cambia ulteriormente con le edizioni a stampa e in particolare dopo l'edizione aldina curata dal Bembo sugli autografi (1501), quando il Triumphus Famae ritornerà ad essere composto soltanto da tre capitoli, il primo dei quali sarà Da poi che morte, per esteso, mentre il capitolo Nel cor sarà posto in appendice oppure semplicemente rifiutato.

Questo schema, a mio avviso utilissimo a chi voglia —o debba- districarsi nella selva del corpus iliciniano, consente infatti di procedere ad una prima collatio, senza l'obbligo di addentrarsi nell'analisi delle singole lezioni: nel caso specifico della traduzione catalana, questa semplice verifica ci consente infatti di stabilire a quale di queste tre fasi redazionali apparteneva il modello che il nostro traduttore aveva sottomano, restringedo così notevolmente il campo d'indagine.

Vediamo brevemente quali capitoli, e in quale ordine, ${ }^{13}$ compongono il nostro testo:

T.Cupidinis I ( 1 )

T.Cupidinis II ( 3 )

T.Cupidinis III ( 4 )

T.Cupidinis IV ( 2)

T.Pudicitie I ( 5)

T.Mortis I ( TM $1 \mathrm{a}+6)^{14}$ Inc. "Quanti già nella ettà matura ed acra»

T.Mortis II ( 7) Expl. «morte bella parea nel suo bel viso»

Inc. «Nel tempo che rinova i miei sospiri» Expl. «De incatenato Giove inanci al carro»

Inc. «Era si pieno il cor di maraviglia» Expl. «Et qual è mèl temperato col assenzio»

Inc. "Poscia che mie fortuna in força altrui» Expl. «che i piè va inanci et l'ochi torno in dietro»

Inc. "Stanco già di mirar non sacio anchora» Expl. «udi cantar ffra l'una e l'altra riva»

Inc. "Quando ad un luogo et ad un tempo quivi» Expl. «ffra quai cognobbe hyppolito et Ioseppe»

Inc. «La nocte che seguì l'orribil caso» Expl. «Tu starai in terra sença me gran tempo»

13. Seguo l'ordine con il quale appaiono nel testo, indicando l'ordine delle edizioni critiche moderne, diverso da quello, tra parentresi tonde.

14. Questo primo capitolo del T. Mortis presenta la fusione del frammento TM 1a, inserito come incipit, con l'originario primo capitolo TM I: vv. 1-18: TM 1a, vv. 1-18;

v. 19: «Lui onde agli occhi mei quel lume naque» (TM 1a, v. 19);

v. 20: «Che oggi è ignudo spirto et poca terra» (TM I, v. 2);

v. 21: «Quella per cui ben far prima mi piacque» (TM 1a, v. 21 );

vv. 22-190: TM I, vv. 4-172. 
T.Famae I ( 1a)

T.Famae II ( 8)

T.Famae III ( 9)

T.Famae IV ( 10)

T.Temporis I ( 11)

T.Divinitatis $^{15}$ ( 12)
Inc. «Nel cor pien d'amarisima dolceça»

Expl. «Poi alla fin Artù re vidi e Carlo»

Inc. «Da poi che morte triunphò nel volto» Expl. «si come adviene a chi virtù relinque»

Inc. «Pien d'infinita e nobil meraviglia» Expl. «magnanimo, gentil, costante e largo»

Inc. «Io non sapea da tal vista levarmi» Expl. «et poi revolsi gli occhi in altre parte».

Inc. «Nel aureo albergo con l'aurora inansi» Expl. «così el Tempo triumpha i nomi e 'l mondo»

Inc. «Da poi che sotto il ciel cosa non vidi» Expl. «esser stato danno e non vantaggio..."

Nel primo tomo, quello di Parigi, sono completi sia i Triumphi che il commento. Il tomo dell'Ateneu di Barcellona, invece, è acefalo e mutilo: del nostro T. Famae I mancano infatti, oltre alla prime carte contenenti l'inizio del commento, anche i 14 versi centrali (vv. 37-50) con il relativo commento, mentre alla fine del codice mancano gli ultimi 28 versi (vv. 118-145) del T. Divinitatis, nonché la porzione corrispondente della glossa iliciniana.

Sapendo dunque che il nostro T. Famae è composto da quattro capitoli, il primo dei quali è Nel cor pien d'amarissima dolcezza e il secondo Da poi che morte, entrambi presenti per esteso, possiamo dedurre che la traduzione catalana dipende da una delle edizioni che appartengono al secondo gruppo (14781500), corrispondente al ramo D dello stemma codicum stabilito dal Wilkins per le edizioni quattrocentesche dei Triumphi. ${ }^{16}$

Sarebbe sufficiente, a questo punto, un esame testuale più approfondito per stabilire con maggior precisione a quale dei tredici incunaboli di questo gruppo corrisponda il nostro testo: purtroppo però la scarsa utilità, almeno per quanto riguarda $\mathrm{i}$ "Triumphi con commento", dello schema delle varianti approntato dall'Appel nella sua edizione critica, ${ }^{17}$ e l'assenza quasi assoluta di

15. Così nel manoscritto, mentre nelle edizioni moderne appare come Triumphus Eternitatis.

16. Ernest Hatch WILKINS, The making of the Canzoniere and other Petrarchan studies, Roma, 1951, p. 387-401.

17. Carl ApPel, Die Triumphe Francesco Petrarcas, Halle: Max Niemer, 1901. A causa di alcuni problemi di tipo metodologico, l'efficacia di questa edizione come strumento per lo studio dei Triumphi commentati risulta infatti seriamente compromessa: innanzitutto non rende conto della presenza del commento, che, come abbiamo detto, costituisce invece una premessa necessaria per l'individuazione del modello; in secondo luogo, vertendo soltanto sui versi poetici, non consente di distinguere con precisione i differenti testimoni fra loro (i tredici incunaboli che formano il gruppo II, infatti, essendo basati — secondo una pratica tipografica allora diffusissima - ognuno sul precedente, e dunque testualmente strettamente dipendenti l'uno dall'altro, non soltanto condividono lo stesso testo ma addirittura, 
strumenti filologici per il testo dell'Ilicino, lo rendono un compito tutt'altro che facile, e apparentemente l'unica soluzione per risalire al testo dal quale dipende la traduzione catalana sarebbe dunque quella di procedere ad una minuziosa collatio dei tredici testimoni.

In realtà, come cercherò di dimostrare a continuazione, la metodologia seguita da Wilkins per analizzare i differenti incunaboli offre degli spunti interessantissimi per semplificare questo processo analitico, permettendoci di ridurre ulteriormente il campo d'indagine senza entrare, almeno per il momento, nel merito delle numerose varianti testuali. Lo studioso, che utilizza gli elementi formali, come la mise en page e la mise en text, in un'ottica filologica (si tratta di quella che oggi si conosce come filologia materiale), prende infatti in considerazione principalmente tre elementi: il tipo di carattere (gothic o roman type), la dimensione dei caratteri (se i versi petrarcheschi ed il commento presentano o no lo stesso modulo) e l'organizzazione del testo poetico (se questo appare in forma di rubrica, se nel verso delle carte è giustificato a sinistra o a destra, se lo spazio laterale è occupato o no dal commento). L'applicazione di questa stessa metodologia ai nostri tredici incunaboli ci consente così di suddividere il nostro corpus in due sottogruppi, ${ }^{18}$ il primo dei quali è costituito dai primi tre testimoni del nostro ramo D, ovvero Ve78, Ve81 e Ve84 (presentano il gothic type, caratteri di dimensioni analoghe per i due testi e le terzine in forma di rubrica, sempre giustificate a sinistra, con gli spazi laterali occupati per il commento), mentre il secondo da Ve86 e da tutti gli altri incunaboli di questo gruppo (si utilizza sempre il roman type e si distinguono i due testi attraverso un modulo maggiore per i versi poetici; gli altri elementi invece non cambiano). Confrontando questi risultati con la mise en page del nostro manoscritto catalano (modulo analogo per i due testi e versi poetici in forma di rubrica, sempre guistificati a sinistra, con lo spazio laterale occupato dal commento), possiamo concludere che questo codice riproduce, in buona misura, la mise en page del primo gruppo: l'unico elemento formale che lo distingue è che il testo poetico, in forma di rubrica, vi appare scritto con inchiostro rosso, mentre il commento con inchiostro nero. Pur non apportando conclusioni definitive, questo procedimento ci fornisce comunque indicazioni preziose, e ci consente, in questo caso, di ridurre ulteriormente il numero (da tredici a tre) dei possibili modelli della nostra traduzione.

Possiamo trovare una conferma della validità di questa metodologia, e più in generale dell'utilità di considerare una determinata mise en page alla stregua di un locus criticus filologico, capace di generare un errore significativo, nel quarto capitolo del T. Famae, dove appaiono infatti due errori la cui genesi deve essere spiegata a partire da un particolare assetto della pagina. Alle cc.

nella maggior parte dei casi, le stesse varianti); finalmente, non prendendo in considerazione la tradizione a stampa, finisce per registrare le varianti di un codice come P7 (Paris, Bibliothèque Nationale. ital. 552), che invece è una semplice copia manoscritta dell'incunabolo bolognese del 1475, prima edizione a stampa del commento iliciniano.

18. D'ora in avanti farò riferimento ai diversi incunaboli utilizzando una sigla composta dalle iniziali del luogo di edizione e dalle ultime cifre dell'anno (Venezia 1478 > Ve78). 
135v e 137v del tomo dell'Atenu osserviamo infatti l'apparente omissione di tre versi (un singolo verso nel primo caso, due nel secondo) dei Triumphi: in realtà i versi si trovano semplicemente inseriti nel testo del commento, come se chi realizzò la traduzione non si fosse accorto del loro particolare status. Nel primo caso il v. 7 "Aristotil poi pien d'alto ingegno" appare ancora in italiano (sottolineato però in rosso, nel tentativo evidente di emendare l'errore), mentre nel secondo i vv. 8-9 "Pictàgoras, que primer umilment / philosoffia nomena per nom digne» appaiono, sempre all'interno del commento, già tradotti, con una nota marginale del XVI secolo che dice "No de Pictagoras» (in realtà i versi ci sono, ma in catalano!). L'origine di questi errori andrà cercata, proprio come se si trattasse di un normale procedimento filologico, in una lezione particolarmente difficile: in questo caso, vista la natura formale degli errori, dovremo supporre una mise en page che, rendendo i versi isolati poco leggibili all'interno della pagina, ne giustifichi la genesi. Il modello della nostra traduzione dovrà dunque essere, necessariamente, uno dei tre incunaboli che compongono il primo sottogruppo (Ve78, Ve81 o Ve84), stampato con caratteri gotici di uguali dimensioni per i due testi; infatti, come abbiamo già detto, Ve86 e tutti gli altri incunaboli del secondo sottogruppo presentano caratteri di dimensioni differenti per i due testi, i quali, anche in assenza di spaziatura, rendono comunque impossibile non avvertire la presenza del verso petrarchesco. Una rapida verifica condotta su Ve78, Ve81 e Ve84 ci permette di constatare che effettivamente in tutti e tre i casi, nelle pagine corrispondenti ai due errori, i versi petrarcheschi appaiono "persi» all'interno della pagina.

Ristretto dunque il gruppo dei possibili modelli a tre testimoni, e malgrado i limiti cui accennavamo sopra (soprattutto a proposito dello schema delle varianti offerto da Appel), la collazione diventa un processo decisamente più agile. Ciò nonostante, poiché mi sembra che supererebbe comunque i limiti di questo intervento, non entrerò in merito ai dettagli dell'analisi filologica, ma mi limiterò ad offrirne direttamente i risultati: la traduzione catalana fu condotta, quasi certamente, sul testo del primo incunabolo di questo gruppo, ovvero quello stampato a Venezia nel 1478 da Reynaldus de Novimagio e Theodorus de Reynsburch. Infatti, mentre l'edizione immediatamente successiva a questa, ovvero Ve81 (Venezia, 1481, Leonardus Wild) presenta una vicinanza tale da poter essere considerata descripta, ${ }^{19}$ l'edizione Ve84 (1484, Venezia, Petrus de Plasii Cremonensis, dictus Veronensis) è invece portatrice - lo vedremo poco oltre- di un buon numero di varianti che la escludono definitivamente quale possibile modello della nostra traduzione catalana.

Lo schema degli errori significativi del testo dei Triumphi che propongo a continuazione, approntato seguendo grosso modo i luoghi stabiliti da Appel, riassume quanto appena detto e illustra - a mio avviso in modo definitivol'identificazione proposta.

19. Questo è un caso esemplare dell'inefficacia dello schema Appel: i due testimoni infatti, proprio in virtù della pratica tipografica cui accennavo sopra, risultano latori delle stesse varianti, ed è praticamente impossibile identificare errori significativi che permettano distinguerli. 


\section{Traduzione catalana}

\section{Venezia 1478}

\section{T.Cupidinis I}

v. 4 «Scaldava il sol già l'un e l'altro corno»

v. 6 "Correa gelata al suo usato soggiorno"

v. 50 "Scoperson quel che 'l viso mi celava"

v. 128 "Quello è Jason, et quell'altra è Medea» v. 4 «Scaldava il sol già l'un e l'atro corno» et $\mathrm{Ve} 84$

v. 6 "Correa gelata al suo usato soggiorno"

v. 50 "Scopersson quel che 'I viso mi celava"

v. 128 "Quello è Jason, et quell'altra è Medea»

\section{T.Cupidinis II}

v. 23 "Che la su casta donna aspecta et priega»

v. 31 "L'altra è Porcia che 'l ferro al foco afina»

v. 145 "Cossì preso mi truovo et ella sciolta"

v. 159 "Come sença morir si muere et langue»

v. 165 «Viver sendo dal cor l'alma divisa»

v. 190 «Et qual è mèl temperato col assenzio»
"Che la sua casta donna aspecta et priega" "L'altra è Portia che "I ferro al foco affina» Altra Ve84

"Così preso mi trovo et ella sciolta" "Come sença morir si muore et langue» "Viver sendo dal chor l'alma divisa" «Et quale è mèl temprato col assentio» Il capitolo termina con questo verso.

\section{T.Cupidinis III}

v. 15 "Con la lingua già stancha la richiama»

v. 27 "Et havea hun suo stile legiadro et raro"

v. 30 "Gente che d'amor givan ragionando" v. 42 "Anchor fa honor col suo dir nuovo et bello" v. 143-145 «Et damnoso guadagno et util danno»

«Et grandi ove più scende chi più sale»

«Stancho riposo et riposato affanno»
"Con la lingua già stancha la richiama» "Et havea un suo stil legiadro et raro" "Gente che d'amor givan ragionando" "Anchor fa honor col suo dir nuovo et bello" «Et damnaso guadagno et util damno» damnoso Ve84

"Et grandi ove più scende chi più sale» Chiaro disonor et gloria oscura et nigra $\mathrm{Ve} 84$ "Stancho riposo et riposato affanno» Perfida lealtade et fido inganno Ve84

\section{T.Cupidinis IV}

v. 6 "Pasavan dulcemente lacrimando»

v. 17 «Chi tu se' inançi, dapoi che sì bene»

v. 49 «Et ben che il fesse onde mi dolse et duole»

v. 186 «Udì cantar fra l'una et l'altra riva»
«Passavan dolcemente lacrimando» "Chi tu se' inançi, da poi che sì bene» «Et benché il fesse onde mi dolse et duole» «Udì cantar fra l'una et l'altra riva»

\section{T.Pudicitie I}

v. 1 "Quando ad un luogo et ad un tempo quivi»

v. 94 "Quivi mille famose et chare salme»

v. 107 "Che sbigottisse, et duolsi accolto in acto" v. 134 "Havieno l'arco la pharetra speçato»
"Quando ad un luogo et ad un tempo quivi» locho Ve84

"Quivi mille famose et chare salme» chiare Ve84

"Che sbigottisse, et duolsi accolto in acto» "Havieno l'arco la pharetra speçato» Et l'arco, et la pharetra havien spezato Ve84 


\section{T. Mortis $\mathrm{I}^{20}$}

v. 145 (v. 164) «Virtù morta et belleça et leggiadria»

"Virtù morta et belleça et leggiadria» legiadria Ve84

\section{T. Mortis II}

v. 114 «Se non s'aita ch'io il cognosco ai segni» v. 124 "Di pocha fede, hor io se no 'l sapessi»" v. 149 «Su gli tuoi i decti te presente accolsi?»
«Se non s'aita ch'io il cognosco ai segni» "Di pocha fede, hor io se no'l sapessi» "Su gli tuoi i decti te presente accolsi?»

\section{T. Famae I}

L'Appel tralascia l'analisi dei luoghi significativi di questo capitolo.

\section{T. Famae II}

v. 13 "Così venia et io di quale schole» v. 88 «Appio conobbi a gli ochi soi che gravi»

v. 113 «Di quel gran nido et Garulo inquieto»

v. 126 "Che hebber non meno il naturale disio»
"Così venia et io di quale schole" "Appio cognobbi agli ochi soi che gravi» a gli $\mathrm{Ve} 84$

«Di quel gran nido et Garulo inquieto» Garrulo Ve84

"Che hebber non meno il naturale disio»

\section{T. Famae III}

v. 76-78 «Poi stendendo la vista quanto io basto» "Rimirando ove l'occhio oltre non varca» "Vidi il giusto Ezechia et Sansone vasto»

v. 113 "Che suo bel viso et la ferrata coma" v. 151 «Quel di lungi seguiva il saladino»
"Poi stendendo la vista quanto io basto" "Rimirando ove l'occhio oltre non varca" "Vidi il giusto Eçechia et Sansone vasto» sanson Ve84

"Che suo bel viso et la ferrata coma" "Quel di lungi seguiva il saladino»

\section{T. Famae IV}

v. 25 «Un gran fulgur parea tutto di foco» v. 26 «Seco era Eschyne che 'l pote sentire» v. 41 «Un che gli ebbe invidia et videl torto» v. 119 "La sua tela gentil ordì in carte» v. 121 «Et poi revolsi gli ochi in altre parte»
"Un gran fulgur parea tutto di foco» «Seco era Eschyne che "l pote sentire» "Un che gli ebbe invidia et videl torto "La sua tela gentile ordì in carte» «Et poi revolsi gli ochi in altre parte»

\section{T. Temporis}

v. 1 « $\mathrm{Ne}^{21}$ aureo albergo con l'aurora inanci»

v. 23 «Et che il mio volo lo radoppi e danni»
«Del aureo albergo con l'aurora inançi» el Ve84

"Et che il mio volo lo radoppi e danni»

20. Per la consistenza di questo capitolo si veda lo schema precedente; l'Appel si occupa soltanto del testo del capitolo Quella leggiadra (TM I), a cui si riferisce l'unico luogo critico che troviamo in questo schema, mentre non commenta il frammento Quanti già (TM 1a). Dato che in questo modo la numerazione dei versi non coincide, essendo il nostro capitolo più lungo di quello dell'Appel, utilizziamo la sua numerazione per il luogo critico, fornendo tra parentesi quella reale del manoscritto.

21. Nel manoscritto la lettura è dubbia, anche se sembrerebbe questa la forma corretta, che infatti troviamo anche in Massó Torrents ( J. MASSÓ TORRENTS, op. cit, p. 60), mentre Romano registra Del (D. Romano, op. cit., p. 15). 


\section{T. Diviniatis}

v. 3 "A me mi volsi et dissi: in che ti fidi?» v. 26 «Vidi in un pie colui che mai non stette» v. 70 "Quasi spianati inanci et drieto i poggi» v. 72 «Nostro sperar o rimembrar s'appoggi»
"A me mi volsi et dissi: in che ti fidi?" "Vidi in un pie colui che mai non stette» "Quasi spianati inançi et drietro i poggi» "Nostro sperar o rimembrar s'appoggi»

Il v. 121 si trovava nella parte del codice oggi perduta.

Come possiamo osservare, la corrispondenza tra i due testi è pressoché totale: dei 49 luoghi critici esaminati, l'unica divergenza filologicamente rilevante è infatti costituita dalla variante secondaria, e oltretutto di dubbiosa lettura, "Nel aureo» per il «Del aureo» che invece Appel mette a testo ( T.T., v.1 «Del aureo albergo co l'aurora inançi»). ${ }^{22}$

Per quanto riguarda il capitolo Nel cor pien d'amarissima dolcezza, tralasciato dall'Appel ma che come abbiamo visto costituisce, di per sé, uno dei principali discrimini tra le diverse fasi redazionali, offrirò invece uno schema basato sull'analisi degli errori che, già presenti nel modello e ripetuti nel manoscritto, costituiscono indizi sicuri di filiazione. La numerazione dei versi, e il testo degli stessi, si riferiscono al manoscritto catalano.

\section{v. 6 «Bursenne lei fra le belle alme lucenti». (Gursene Ve78 Girsene Ve84)}

Si tratta di un errore per il verbo Girsene. La lezione del manoscritto è il risultato, evidentemente a partire dalla forma Gursene, della confusione, costante nel manoscritto, tra il carattere $G$ e $B$ ( $c f r$. l'errore ricorrente Bueo per Gneo Pompeo).

vv. 22-23 «Et da man dextra havea quel gran romano che fe' in germania e ffrancia tal ruhina».

Pur non costituendo un errore vero e proprio, questi versi rappresentano, come abbiamo già accennato, il discrimine tra i testimoni del primo e del secondo gruppo: l'incunabolo Ve78 e tutte le successive edizioni di quel gruppo (e dunque sia Ve81 che Ve84) presentano infatti i capitoli Nel cor e Da poi per esteso, mentre nel gruppo facente capo a Bo75 il capitolo Nel cor è completato dai versi di Da poi che morte («Et da man dextra havea Cesare e Scipio»).

v. 83 «e doi gran scloion che Spagna oppresse»

(scloion Ve78 Scipion Ve84)

v. 129 «similmente da l'un lalto fosco»

(lalto Ve78 lato Ve84)

22. Il discrimine principale tra le varianti 1 e 2 risede infatti, secondo Appel, nell'alternanza tra «Del aureo» e "Del taureo», rispettivamente. 


\section{v. 138 "Chiari per sé ma più per chi ne scuisse» \\ (scuisse Ve78 scrisse Ve84)}

\section{v. 141 «Hemici prima, amici poi sì fidi» (Hemici Ve78 Nemici Ve84)}

La prima conseguenza di questa identificazione consiste, naturalmente, nella possibilità di stabilire un termine post quem per la stesura dell'opera, restringendo così notevolmente la datazione della traduzione, da un generico "darreries del XV ${ }^{\text {en }}$ segle» ${ }^{23}$ a post 1478 , data di pubblicazione del modello italiano.

Per quanto riguarda poi un eventuale limite cronologico ante quem, mi sembra che gli spunti più interessanti vengano dall'analisi delle traduzioni castigliane di Antonio de Obregón e Hernando de Hozes. Questi autori, secondo quanto essi stessi dichiarano nelle prefazioni delle rispettive traduzioni, consideravano infatti già nella prima metà del Cinquecento il testo del nostro incunabolo superato e scorretto. Vediamo i passi in questione.

Antonio de Obregón, nella sua traduzione pubblicata a stampa nel 1512, afferma a proposito del testo dei Triumphi:

Mas como Francisco Petrarca los compuso siendo ya de mucha edad no pudo quedalle tiempo para emendallos como en los Petrarcas viejos se paresce, mas porque la doctrina de varon tan excelente no quedase alli viciosa fue en Venecia cometida esta obra a persona tan abundante de letras que puso los seys triumphos como quien los escrivio los pusiera si la vida le durara.

E aggiunge, a proposito del nostro capitolo Nel cor pien d'amarissima dolceça:

$Y$ el triumpho de la Fama contiene tres capitulos solamente, que el que ponen por primero se convierte en segundo como mas claro paresce en los Petrarcas sin comento emendados, y no difieren en cosa más que en la orden del poner las personas. ${ }^{24}$

Innanzi tutto osserviamo che l'Obregón, evidentemente in data anteriore alla pubblicazione della traduzione (1512), conosce l'operazione filologica bembiana (è lui la "persona tan abundante de letras») e che considera il testo dei Triumphi «como en los Petrarcas viejos se paresce» certamente scorretto. Converrà dunque iniziare dal chiedersi cosa intenda per «viejos». Nonostante fosse al corrente del lavoro del Bembo sugli autografi petrarcheschi, non ritengo plausibile che possa riferirsi a codici del XIV secolo, e dunque contemporanei o di poco posteriori al Petrarca; credo invece che per Petrarcas viejos intenda 
quei manoscritti o incunaboli, come il nostro, dell'ultimo quarto del XV secolo, magari ancora in caratteri gotici e, come si evince dalla seconda citazione, contenenti un testo ormai evidentemente superato; la redazione che il nostro traduttore castigliano considera corretta, e che infatti traduce, comporta che il T. Famae contenga «tres capitulos solamente», mentre sappiamo che il nostro manoscritto catalano, e tutte le edizioni quattrocentesche di questa famiglia, ne hanno quattro. ${ }^{25}$

Nella seconda traduzione in questione, quella di Hernando de Hozes, stampata per la prima volta a Medina del Campo nel 1554, e dunque successiva alla sostituzione del commento iliciniano con quello del Vellutello (1525), l'autore afferma:

En un libro de los Triumphos de Patrarcha de impression antigua que yo tengo comentado de Bernardo Ilicino ay en el triumpho de la Fama de que ahora queremos tratar un capitulo puesto antes que los tres que aqui se veran, el qual se dexa de poner porque en los que andan glossados por Alexandro Vellutello que yo he visto no ay mas destos tres capitulos ni aun tampoco en la traduccion hecha en nuestra lengua, aunque sea verdad que en algunos de los de nueva impresion he visto este capitulo puesto por su parte y sin glossa al fin de todas las obras con solamente titulo que dize capitulo de Francisco Petrarcha. ${ }^{26}$

L'Hozes si riferisce certamente, parlando nel 1554 di un libro di Petrarca di " impression antigua», commentato da Bernardo Ilicino, ad uno degli incunaboli di fine Quattrocento dei Triumphi, e lo conferma il fatto che in questo libro il T. Famae abbia ancora quattro capitoli, mentre già nella traduzione "hecha en nuestra lengua», cioè quella di Obregón, ne vengono accettati soltanto tre; inoltre, visto che quest'ultima traduzione, del 1512, non rientra nel concetto di «antiguo», questo aggettivo non potrà che essere riferito ad un testo, come il nostro incunabolo, precedente di diversi anni a quella data. Il «capitulo puesto antes que los tres» è il nostro capitolo Nel cor pien d'amarissima dolceça: rifiutato già nelle edizioni dei primi decenni del XVI secolo, riapparirà poi nelle edizioni «de nueva impression", della metà del Cinquecento appunto, come capitolo sciolto, in appendice alla fine del libro.

Mi sembra dunque piuttosto evidente che il nostro testo non può essere datato oltre i primissimi anni del XVI secolo; infatti, qualora fosse della prima metà del XVI secolo, ovvero contemporaneo o posteriore a quello d'Obregón, dovremmo supporre che il traduttore catalano utilizzasse un modello ritenuto già obsoleto dai suoi contemporanei. Pur non essendo teoricamente impossi-

25. I tre capitoli del T.Famae in Obregón sono: TF I: «Despues que muerte triunpho | de quien vençerme solia...»; TF II: «Hizome marauillado | el pueblo muy excelente... »; TF III: «Yo quitarme de tal vista | no supe hasta que oy... »; il capitolo assente, come nelle edizioni cinquecenteche, è il nostro Nel cor pien d'amarissima dolceça.

26. Hernando de HOZEs, Los Triumphos de Francisco Petrarcha, ahora nuevamente traduzidos en lengua castellana..., Medina del Campo, 1554. Le citazioni sono dall'edizione del 1581; il corsivo è nostro. 
bile, ritengo questa eventualità francamente poco probabile. Credo che si possa quindi concludere, con buona approssimazione, che la stesura della traduzione catalana del commento di Bernardo Ilicino ai Triumphi, basata sul testo dell'incunabolo veneziano del 1478 , dovrà risalire al periodo compreso tra il 1478 , data di pubblicazione del modello italiano, e il 1512, data di pubblicazione della traduzione di Obregón.

Pur non addentrandoci in una descrizione codicologica esaustiva del manoscritto, converrà infine osservare che tutti i dati materiali identificabili con certezza, come ad esempio il tipo di carta, sembrano confermare questa datazione. La filigrana della mà amb la stela presente nella maggioranza delle carte del tomo di Barcellona, ${ }^{27}$ certamente di origine italiana (importata a València da mercanti genovesi, probabilmente procedente dal mulino di Voltri o dal savonese), corrisponde infatti a quella utilizzata, attorno alla metà degli anni settanta del XV secolo, per la stampa di alcuni tra i primi incunaboli valenzani. ${ }^{28}$

27. Anche se le descrizioni del ms. II dell'Ateneu vi identificano solo la mà am la estrella (mentre per il tomo parigino solo la mà amb flor, che purtroppo non ho potuto esaminare), credo che nel codice dell'Ateneu vi appaiano invece due filigrane distinte, la mà amb la estrella (cinque punte) e la mà amb la flor (con sei punte o petali); a sostegno di questa proposta si veda l'atto notarile: «En 25 de Novembre de 1478 los hermanos Berniçó, mercaderes de Génova, venden al Magnífico Miquel del Miracle, de València, 200 balas de papel de la mano y la estrella, 108 balas de la mano y la flor, al precio de cinco libras, moneda real de València, por cada bala, unas con otras", da Oriol VALLS I SUBIRÀ, El papel y sus filigranas en Catalunya (Paper and watermarks in Catalonia) 2 vol., Amsterdam: Paper Publications Society, 1970. Il Serrano Morales, nel suo Diccionario de las imprentas, alla voce Lamberto Palmart, p. 432-455, distingue tra la mano con la estrella a cinque punte e quella a sei punte, quest'ultima corrispondente con quella che noi invece consideriamo come flor.

28. Ritroviamo questa stessa filigrana (come abbiamo visto, addirittura importata dagli stessi mercanti italiani) in ben tre opere tra quelle attribuite al tipografo tedesco Lambert Palmart, stampate a València attorno al 1474-75: Les Trobes en lahor de la Verge Maria (1474), il Comprehensorium (1475) e le Obras de Salustio (1475). 